\title{
BMJ Open Exploratory qualitative study to understand the underlying motivations and strategies of the private for-profit healthcare sector in urban Bangladesh
}

\author{
Alayne Mary Adams, ${ }^{1,2,3}$ Rushdia Ahmed, ${ }^{\circledR 2,3}$ Tanzir Ahmed Shuvo, ${ }^{4}$ \\ Sifat Shahana Yusuf, ${ }^{3}$ Sadika Akhter, ${ }^{3}$ Iqbal Anwar $^{3}$
}

To cite: Adams AM, Ahmed R, Shuvo TA, et al. Exploratory qualitative study to understand the underlying motivations and strategies of the private for-profit healthcare sector in urban Bangladesh. BMJ Open 2019;9:e026586. doi:10.1136/ bmjopen-2018-026586

- Prepublication history and additional material for this paper are available online. To view these files, please visit the journal online (http://dx.doi. org/10.1136/bmjopen-2018026586).

Received 10 September 2018 Revised 17 April 2019 Accepted 31 May 2019

Check for updates

(C) Author(s) (or their employer(s)) 2019. Re-use permitted under CC BY-NC. No commercial re-use. See rights and permissions. Published by BMJ.

${ }^{1}$ Department of International Health, Georgetown University, Washington, District of Columbia, USA

${ }^{2}$ BRAC James P Grant School of Public Health, BRAC University, Dhaka, Bangladesh

${ }^{3}$ Health Systems and Population Studies Division, icddr,b, Dhaka, Bangladesh

${ }^{4}$ Department of Health Services Policy and Management, University of South Carolina, Columbia, South Carolina, USA

Correspondence to

Rushdia Ahmed;

ahmed.rushdia@yahoo.com

\section{ABSTRACT}

Objectives This paper explores the underlying motivations and strategies of formal small and mediumsized formal private for-profit sector hospitals and clinics in urban Bangladesh and their implications for quality and access.

Methods This exploratory qualitative study was conducted in Dhaka, Sylhet and Khulna City Corporations. Data collection methods included key informant interviews (20) with government and private sector leaders, in-depth interviews (30) with clinic owners, managers and providers and exit interviews (30) with healthcare clients.

Results Profit generation is a driving force behind entry into the private healthcare business and the provision of services. However, non-financial motivations are also emphasised such as aspirations to serve the disadvantaged, personal ambition, desire for greater social status, obligations to continue family business and adverse family events. The discussion of private sector motivations and strategies is framed using the Business Policy Model. This model is comprised of three components: products and services, and efforts to make these attractive including patient-friendly discounts and service-packages, and building 'good' doctor-patient relationships; the market environment, cultivated using medical brokers and referral fees to bring in fresh clientele, and receipt of pharmaceutical incentives; and finally, organisational capabilities, in this case overcoming human resource shortages by relying on medical staff from the public sector, consultant specialists, on-call and less experienced doctors in training, unqualified nursing staff and referring complicated cases to public facilities.

Conclusions In the context of low public sector capacity and growing healthcare demands in urban Bangladesh, private for-profit engagement is critical to achieving universal health coverage (UHC). Given the informality of the sector, the nascent state of healthcare financing, and a weak regulatory framework, the process of engagement must be gradual. Further research is needed to explore how engagement in UHC can be enabled while maintaining profitability. Incentives that support private sector efforts to improve quality, affordability and accountability are a first step in building this relationship.
Strengths and limitations of this study

- This exploratory study is among the first in Bangladesh to query the underlying motivations and strategies of the urban private for-profit sector.

- The study employs qualitative methods to enable indepth understanding of factors influencing healthcare practices as reported by private facility owners and providers.

- A limitation of the study was the unwillingness of certain respondents to disclose strategies they employ to grow and flourish their private sector business.

\section{INTRODUCTION}

The Sustainable Development Goal 3 (SDG3) of reaching universal health coverage (UHC) by 2030 is challenging in pluralistic healthcare systems such as Bangladesh. ${ }^{1}$ The country is also urbanising rapidly as reflected in an average urban population growth rate of $3 \%$ per year and an astonishing $7 \%$ per year in poor informal settlements. ${ }^{23}$ If these trends continue, by 2040, over half of Bangladesh's total population will reside in urban areas. ${ }^{4}$ In this context, challenges to achieving UHC are amplified as demand for services increases, and the healthcare market shifts towards the private sector. ${ }^{56}$ In Bangladesh, reaching the SDG target 3.7-ensuring every person has access to affordable quality healthcare services, including financial risk protection-is particularly daunting given that $67 \%$ of national health expenditure is already out of pocket. ${ }^{7}$ However, Bangladesh is also a country that routinely defies expectations, witnessed in its spectacular health achievements over the last four decades in terms of reductions in total fertility rate and rates of maternal, infant and childhood mortality. ${ }^{8}$ Although some of this success may be due to coverage of publicly financed free services, ${ }^{9}$ 
and its role in reducing use-inequities, ${ }^{10}$ the contribution of private healthcare sector remains poorly understood. This understanding is complicated by the widespread phenomenon of dual practice, whereby a large proportion of public sector providers are also involved in private practice to supplement income. ${ }^{11}{ }^{12}$ Given its primary for-profit orientation, there is widespread scepticism about the potential contribution of the private sector towards UHC. In the absence of strong regulatory capacity on the part of the state, these concerns relate to inappropriate or unnecessary care, inequitable access due to escalating costs and poor quality of care. As a result, the public sector continues to be the major focus of Government efforts towards UHC targets. ${ }^{13}$

However, recent data suggest that neglect of this sector is short sighted. For example, the 2016 Bangladesh Maternal Mortality Survey (BMMS) shows that for obstetric complications, only $25.5 \%$ of women visited public sector facilities and the rest used some kind of private facility or informal provider as a first source of care. ${ }^{14}$ The use of the private sector for delivery services is also increasing. According to BMMS data, only $2.6 \%$ of mothers delivered in private sector facilities in 2001, rising to $11.3 \%$ in 2010 and $29 \%$ in 2016, whereas public sector delivery trended from $5.8 \%$ (2001) to $14 \%$ (2016) over the same period. ${ }^{14}$ The contribution of the private sector to the explosive growth of C-section delivery is particularly concerning. According to 2011 Bangladesh Demographic and Health Survey data, more than half of all C-sections took place in private sector facilities, and among deliveries occurring in private facilities, $72 \%$ were by C-section. ${ }^{15} \mathrm{~A}$ nationwide rise in $\mathrm{C}$-section rates from $17 \%$ in 2011 to $23 \%$ in $2014^{16}$ implies that the private sector share is continuing to increase.

In Bangladesh, the private for-profit sector in health consists of profit-oriented businesses that charge healthcare consumers above actual service costs. The sector includes a heterogenous set of providers that vary in the degree to which they operate within or outside the purview of regulation, registration or oversight by government or professional bodies or possess formally recognised training. At one end of this spectrum are formal private for-profit hospitals and clinics offering diagnostics and both general and specialised medical treatment including surgical procedures, while at the other, unqualified doctors or drug sellers purvey pharmaceuticals whether needed or not. ${ }^{1317}$

In urban areas, the density of private sector services is remarkable. Unlike rural Bangladesh where government healthcare infrastructure is available at district, subdistrict and community levels, in urban areas, the public health system is limited to a handful of poor functioning urban dispensaries and secondary and tertiary hospitals operated by the Ministry of Health and Family Welfare. Designated responsible for urban primary healthcare yet lacking implementation capacity, the Ministry of Local Government has contracted out primary healthcare services to non-governmental organisations (NGOs) on a project basis ${ }^{18} 19$ with a predominant focus on maternal and child health. ${ }^{20}$ Filling the gap in public primary services is the urban private sector that accounts for over $90 \%$ of healthcare facilities (online supplementary annex 1) in urban areas. ${ }^{20}$ Nationally, data from the Directorate General Health Services show an increase in the number of registered private for-profit facilities from 1038 in 2007 to 5023 in 2017, ${ }^{21} 22$ reflecting both a rise in demand for services and the inability of the public sector to generate sufficient supply on its own. Yet even within so-called formal facilities, irregular practices are common. For example, recent evidence from a comprehensive mapping of all health facilities in Sylhet City Corporation found that $40 \%$ of private clinics, hospitals and diagnostic centres had not fully complied with annual registration requirements. ${ }^{23}$

Despite the size of the urban private for-profit sector and its critical role in health service delivery in Bangladesh, relatively little is known about its underlying motivations and business strategies. In this study, we focus on small and medium-sized formal private for-profit health facilities (between 10 and 150 bed capacity) given their substantial urban presence. In Dhaka alone, they represent about $55 \%$ of all hospitals and clinics, with public and NGO sector facilities accounting for the remaining $19 \%$ and $26 \%$, respectively. ${ }^{20}$ With the broader goal of enabling UHC in urban areas, our objectives are: (1) to explore the underlying motivations of owners, managers and providers entering into and sustaining activities in the small and medium-sized formal for-profit private healthcare business and (2) to understand how the business strategies and incentives governing the small and medium-sized formal for-profit private sector enable or hinder quality and financial access.

To frame our exploration of business motivations and strategies, we draw on the Business Policy Model $(\mathrm{BPM}),{ }^{24}$ the basic concepts of which still undergird the logic of current corporate strategy analysis. ${ }^{25} \mathrm{BPM}$ is made up of three basic elements-products/markets, the market environment and organisational capabilities-that interact to determine how a private sector business performs. Specifically, the model specifies how the financial success of a particular good or service offered by a private sector business is a function of its alignment with the market environment and the organisation's capabilities. In the case of healthcare provision, the goal of the private for-profit sector is to ensure that its products or services constitute the most 'profitable value-proposition' in the current market environment, that the market shows sufficient long-term demand for those services and that the services offered align with organisational capabilities to add value. Based on this framework, we will consider how urban small and medium-sized private owners, managers and providers strategise around products, markets and capabilities to ensure success in sustaining and growing their healthcare business. Of particular interest is how these strategies impact quality and access by the urban poor. 


\section{METHODS}

\section{Study design}

This exploratory qualitative study was conducted in three city corporations in Bangladesh and involved interviews with government and private sector leaders, formal private for-profit healthcare actors and consumers of these services.

\section{Study site}

Three cities were purposively selected to capture a wide range of performance on key indicators of healthcare access and utilisation such as rates of vaccination coverage, Antenatal Care (ANC) coverage, child mortality and maternal mortality. ${ }^{15}$ These were: Dhaka, the national capital of Bangladesh, and among the fastest growing megacities in the world; Khulna, a divisional capital located in a district considered high performing in terms of key health indicators; and Sylhet, a divisional capital in one of the poorest health-performing districts in the country. Within each of these cities, we focus individuals involved in small to medium-sized licenced and registered (as reported) private for-profit healthcare businesses (10-150 beds) in the roles of owner, manager, healthcare provider and patient.

\section{Study methods and sampling strategy}

A total of 80 respondents were interviewed from September 2013 to March 2014. In each city, key informant interviews (KIIs) were conducted with a purposive sample of local officials from the Ministry of Health, the Bangladesh Medical \& Dental Council, the Bangladesh Medical Association, the pharmaceutical industry as well as members of the Private Clinic Owner's Association. At the end of each KII, respondents were asked to recommend the names of small and medium-sized private clinic owners and providers who might be willing to participate in in-depth interviews (IDIs). This snowball sampling method helped identify potential respondents working in a sector that is otherwise difficult to access. IDI respondents included private healthcare facility owners, managers and providers. Using a purposive sampling strategy, inpatient and outpatient exit interviews were also performed to document client experiences in the same

Table 1 Types and numbers of interviews conducted in each study site

\begin{tabular}{|c|c|c|c|c|}
\hline Types of respondents & Dhaka & Sylhet & Khulna & Total \\
\hline Key informants & 5 & 7 & 8 & 20 \\
\hline $\begin{array}{l}\text { For-profit health facility } \\
\text { owners and managers }\end{array}$ & 5 & 5 & 6 & 16 \\
\hline For-profit service providers & 4 & 5 & 5 & 14 \\
\hline $\begin{array}{l}\text { Exit interviews with } \\
\text { inpatients }\end{array}$ & 5 & 5 & 5 & 15 \\
\hline $\begin{array}{l}\text { Exit interviews with } \\
\text { outpatients }\end{array}$ & 5 & 5 & 5 & 15 \\
\hline Total & 24 & 27 & 29 & 80 \\
\hline
\end{tabular}

facilities in which IDIs were conducted. Table 1 displays the types and numbers of interviews conducted in each study sites.

\section{Data collection}

Guided by two supervisors with extensive field experience and expertise in qualitative methods and analysis, data collection was performed by 12 social science researchers. Semistructured KII and IDI guidelines were prepared for different groups of respondents. KIIs explored urban health challenges; the range of private care providers and services provided; quality of care, dual practice and referral mechanisms; as well as known strategies to maintain profitability including incentives provided by pharmaceutical companies. IDIs with private healthcare owners, managers and providers considered their underlying motivations in choosing and remaining in the sector; services provided and available human resources; linkages with other formal providers, pharmaceutical representatives and brokers; typical referral mechanisms; sustainability and quality of care practices; and challenges and suggestions for better coordination with the public sector.

Exit interviews elicited narratives concerning the experience and satisfaction of healthcare consumers frequenting private sector facilities in terms of the quality and affordability of services received and whether they intended to return to the same facility in future. In each city, senior researchers tapped into existing networks to identify a number of well-positioned key informants for interview, many of whom provided support in identifying respondents and facilitating access. These existing relationships were crucial to entrée, rapport building and trust in a sector that is typically closed to outsiders.

Two or three researchers were involved in each interview: a facilitator and one or two note-takers. In addition to detailed hand-written field notes, interviews were recorded digitally. Recordings were transcribed verbatim into Bangla as soon as possible following data collection, and field notes and observations were written up in the same time frame. Bangla transcripts were translated into English by skilled translators, and a subsample of translated transcripts were reviewed and back translated by senior researchers to cross-check data fidelity.

\section{Data analysis}

Framework analysis was performed using codes and data displays to systematically examine emerging patterns and themes. ${ }^{26} \mathrm{~A}$ team approach to analysis was employed to minimise individual bias with multiple analysts involved in coding and interpreting data. To begin, each transcript was coded independently on hard copy by two researchers. Initially, seven 'a priori codes' were defined and later inductive codes were also included in the coding framework. After assessing intracoder and intercoder reliability by having two analysts independently code the same sections of text, codes were applied by the research team using Atlas-ti. Code reports were generated 
based on 'a priori' themes and other inductive codes to facilitate the identification of patterns and themes. Data displays were also used to visualise patterns across categories and concepts, and permit systematic analysis.

\section{Ethical considerations}

Prior to interview, written informed consent to participate in the study was obtained from each respondent, as well as permission to be audio-recorded. All elements of consent were described to study respondents orally to clarify the purpose of the research, the measures undertaken to ensure confidentiality and their right to withdraw from the interview at any time for any reason. Arrangements for the place and time of interview were organised in advance according to the respondent's convenience and privacy.

\section{Patient and public involvement}

The research questions and outcome measures of this study were identified with the participation of a technical advisory group composed of formal healthcare owners, managers and providers, policy makers and academics. Neither patients nor the public were involved in study design. Patient involvement was limited to participation in exit interviews that captured their experiences and satisfaction with care received from formal private for-profit clinics. Study findings were shared and discussed through a series of dissemination workshops involving international and bilateral donors, researchers, government officials from the Ministry of Health and Family Welfare involved in hospital services management, planning and quality improvement, as well as leaders from professional medical, nursing and private clinic associations.

\section{RESULTS}

\section{Motivations of private healthcare owners, managers and} providers

To understand the motivations underlying the involvement of private sector actors in the healthcare market, respondents were asked to describe how they came to be involved in the sector and their reasons for sustaining their business. Two groups of motivational factors emerged from analysis: financial and personal.

The monetary rewards associated with running a successful healthcare business were a strong pull factor motivating entry into the private sector. The industry was widely perceived as financially lucrative and therefore an appealing professional choice. Formal for-profit business owners described how profit is a central motivation and that the provision of quality care is critical to ensuring 'good' business, that is, financial gain. One doctor in Sylhet explained, 'the main reason I entered the private sector was business. I worked in a small town near Dhaka city. 55-60 private clinics are there in that small town. All are running well, also gaining profit...' They also explained how profit was generated when necessary services are provided that the public sector is unable to furnish due to insufficient capacity.

There was also a general assumption that working as a doctor in the private sector ensured financial security given the rising demand for services in urban areas. Many providers further described how the opportunity for a stable income for themselves and their families was a key reason for joining and remaining in the profession. According to one private provider in Khulna: 'The thing that attracted me to this profession is financial solvency... I assumed that I will have a superior financial status and I (sustained) that by joining this profession'.

In addition to financial interests, personal motivations also played a role in entering the private healthcare profession. First among these was the desire to furnish needed services to the public and the personal fulfilment that this yields. A number of respondents noted a particular concern for the poor, the vulnerable and the disadvantaged who frequently lack access to quality care. For these providers, a commitment to rectify these inequities was identified as an impetus for entering the private healthcare sector and a reason for continuing their professional engagement.

At the other end of the spectrum was personal ambition. For some respondents, the desire for social status associated with a career in healthcare, and conferred to specialist doctors in particular, was an important factor motivating their decision to engage in private sector practice. Others indicated their aspirations to be part of a challenging, fast-growing profession. Frequent reference was also made to the expectations and ambitions of family members. Pressures from family to pursue private medical practice were widely cited, largely due to the perceived status and income it commands, or because an existing family business needed to be sustained. As one provider from Khulna explained: 'This business is in our family. The forefathers of my father used to run it, after them, my father. I practiced with my father for a very long time, then after (his) death I took charge'.

In a number of instances particular personal circumstances compelled entry into the private healthcare market. One clinic owner and provider described his decision to start a clinic following his mother's death and his desire to do something concrete in her memory: 'When I started my fourth year of medical school, my mother died. At that time, I couldn't take care of my mother due to my studies. I established this clinic in (her) name'. Similarly, a clinic manager in Dhaka claimed that an experience with poor quality healthcare prompted the creation of the facility in which he worked: 'Our Director's child died in a renowned hospital of Bangladesh ... because of the carelessness of the doctors and nurses. So, our Director decided to build a NICU where patients will not face such kinds of carelessness'.

Interesting in these narratives was the spectrum of reasons why our respondents came to be involved in the private healthcare sector ranging from the profit-seeking motivations we typically associate with the sector to a desire to serve the public. Recognising this complexity of 
motivations helps clarify the strategies the sector employs in maintaining their healthcare business and how they might be leveraged to increase access to the urban poor.

\section{Business strategies of private healthcare owners, managers and providers}

Private formal health sector actors reported multiple strategies to ensure business success and sustainability. These strategies helped them increase client flow and satisfaction and derive profits out of the services provided. Adapting the BPM to the context of private sector healthcare, we consider these strategies under the broad headings of products and services, the market environment and organisational capabilities. A particular interest in this exploration is how strategies in these areas can either facilitate or hinder access by the urban poor.

\section{Products/services}

A number of strategies were identified whereby for-profit healthcare businesses aimed to make their services more appealing to both current and potential clientele. One such strategy was the use of patient discounts. The majority of private practitioners interviewed reported using discounts to entice new patients and to reward existing client loyalty. However, philanthropic motivations were also common, with many private sector clinics offering discounts to allow poorer patients access to services they otherwise could not afford. As one private sector provider explained:

There is not a fixed percent, but they do so according to the (financial) state of the patient... Normally we grant $15 \%$ discounts for tests and $10 \%$ for the bed rent. Sometimes we have to give more - even above $50 \%$.

Another widespread strategy was the provision of health packages that bundled services and products together at a fixed price for procedures such as C-section and appendicectomy surgery. This decreased costs to patients compared with the cumulative price of individual services and, in some instances, created opportunity for negotiation between clinics and clients. While this practice was reported to increase patient flow, in certain instances, they had detrimental financial consequences especially when patient complications required additional, unanticipated tests and procedures that the package did not include, yet the clinic was obliged to cover.

Finally, almost all private providers mentioned the importance of cultivating a positive and trusting doctorpatient relationship as crucial to ensuring client loyalty and continuity of care. Respondents described efforts made within the clinical setting to make patients feel valued and comfortable, recognising that client perceptions of provider behaviour and attitudes are important determinants of whether they adhere to treatment or return for subsequent visits. As explained by a doctor in Sylhet:
While the first aim is that the patient gets cured, my behaviour is also important... We, the doctors... tell our students that not all diseases are organic. Some are psychosomatic (and hence) our behaviour with patients is a major factor in providing care. The patient (must) have faith in a doctor that he will be cured ... The doctor must create such faith through conversations and discussion time (with patients) ...

Our findings revealed that positive perceptions of provider conduct were not contingent on the duration of wait time for consultation, nor the length of the provider-patient interaction. Interviews with patients exiting private facilities revealed that even though average consultation times were only $6-7 \mathrm{~min}$ in length and wait times varied between $5 \mathrm{~min}$ and 2 hours and 30 min, reports of patient satisfaction with private sector services were uniformly positive. Many noted their provider's efforts to make them feel comfortable and the quality of services received. As described by one patient leaving a private clinic in Khulna: 'The quality of service is good here... much better than other facilities. The (doctor's) behaviour is very good... he examined me carefully, the nurses, duty doctors and the doctor visited me regularly...'.

The provision of extended service hours was another strategy that was widely perceived to offer a competitive advantage over the daytime operations of NGO clinics by offering greater access to the working population.

\section{Market environment}

A number of strategies were used to maintain market position and cultivate demand for private sector services. Among these was reliance on medical brokers or Dalals as a means to ensure patient flow. Employed by many private healthcare facilities to divert or convince clients to use their services, Dalals typically operate near the entrance of public hospitals or in areas of the city where new migrants to the city first settle. A number of respondents also indicated that informal providers such as drug sellers and unlicenced or 'village' doctors may also act as Dalal for formal private clinics, receiving payment according to the number of patients referred, or in other cases, a percentage of service charges. A private clinic manager from Dhaka explained the importance of this strategy:

Many patients are referred (through agents or brokers). Relatives of a patient who have received services from us (in the past), may also increase publicity. (In return) we give them services at low cost.

Public sector providers are similarly known to act as middlemen, referring patients to their own private practices or those in which they are shareholders. In other instances, providers described referring patients to other private facilities and, like Dalal, receiving a commission for referral. While some of these referrals may be clinically indicated, a number of stakeholders reported that this practice of 'referral for commission' was widespread among private sector doctors, diagnostic centres 
and clinics and used to boost revenue through collusion. A top-ranked government health officer in Khulna described the involvement of doctors in referral and the financial benefits that are accrued:

Suppose, I am an owner of a diagnostic centre. Many brokers are available to me. I will tell them to collect patients from wherever they can, (and) they will be given a percentage. If, a doctor sends patients to me for pathology (testing), I will give him a $40 \%$ or $50 \%$ commission. If I get 2000 taka (USD 23.72) by doing the pathology, then 1000 (USD 11.86) taka is for me and the other 1000 (USD 11.86) is for the doctor the doctor is happy, and so am I. If 10 patients are sent daily, he will receive 10,000 BDT (USD 118.59). (Likewise), if I refer patients to the doctor's facility, he will send patients to my diagnostic service for tests.

Although these practices were perceived to increase patient flow, several stakeholders noted how referrals orchestrated by brokers may be disadvantageous to the urban poor. Diverting patients away from free government services towards private sector providers, brokers effectively increased the cost of care and the patient's financial burden and even more when treatment is unnecessary.

Another practice that nurtures the market environment for private sector services was the close relationship with pharmaceutical companies. Nearly all private practitioners described regular visits from pharmaceutical representatives on a monthly, weekly or even daily basis with the purpose of marketing their products. They also reported receiving incentives to buy and prescribe certain drugs, although this practice is prohibited by the Government's Code of Pharmaceutical Marketing Practices, which states that "no gift or financial inducement shall be offered or given to members of the medical profession for purposes of sales or promotion' ${ }^{27}$ Incentives in the form of money, drug samples and gifts were received routinely, and many admitted their influence on prescription patterns. As one clinic owner explained:

... month after month, different companies pay doctors for recommending their medicines... even giving cash...now if doctors recommend (these drugs), we have little choice (but to keep them in our store)...

Another provider from Sylhet described how the ubiquitous presence of pharmaceutical representatives had effectively changed his prescription practices:

I generally prescribe those medicines which work effectively; still there are some influences such as medical representatives (who) come frequently. They come in the morning, in the evening, automatically we need to keep their medicines ...we use those, prescribe those, but we don't (always) get to check ourselves whether they work or not.

This strategy of cultivating and sustaining provider loyalty is carefully calculated, as one pharmaceutical representative in Khulna recounted:
I see which pharmaceutical company's medicine the doctor is prescribing... We get data from different sources. Some are paid 100000 taka (USD 1185.95) annually, or 3000 (USD 35.58) to 5000 taka (USD 59.30) monthly... then I request him (doctor) to kindly give me a chance and make him a monetary offer. If he agrees then I provide him the agreed amount monthly or yearly. Then he writes our drug in the prescription.

A number of key stakeholders expressed concern about the consequences of pharmaceutical influence on patients, including the development of antibiotic resistance and financial impoverishment by obliging patients to purchase expensive and sometimes unneeded medicines. Private sector providers were also aware of the negative consequences of aggressive pharmaceutical marketing such as the prescription of low-quality drugs, and some expressed concern that decisions about what drugs to stock may be determined by price and discounts received and not what is best for the patient in terms of treatment efficacy. As one pharmaceutical company manager explained, sometimes drugs purveyed by pharmaceutical reps are not even intended for the local market:

Sometimes they (medical representatives) they sell a product not meant for local sale to medicine shops at a discounted rate $[\ldots]$ then motivate drug sellers and doctors not to sell another company's product as they will not get any benefit from them.

Interestingly, several private sector providers justified their relationship with pharmaceutical representatives by explaining their practice of passing on free drug samples to patients with less capacity to pay-for prices well below standard rates.

\section{Organisational capabilities}

Many respondents noted the continual challenge of ensuring adequate, trained human resources in an increasingly competitive urban healthcare market. A consequent practice by private clinics was the use of medical staff from the public sector as consultants for specialist and general services. Duty doctors were most often medical staff with less experience, including honorary trainees, postgraduate medical students and occasionally interns. As office hours in public hospitals typically extend from 08:00 to 14:00, in theory, public sector doctors that engage in private sector practice are only available later in the day. As one clinic owner from Sylhet noted, staff shortages and the provision of specialised services in particular were therefore limited during morning hours: 'The consultants are mostly from the public medical college. So, we face this (doctor shortage) problem from 8:00am-3:00pm.'

Other respondents noted that the practice of public sector doctors attending patients at private facilities during office hours was not uncommon, with a clinic manager from Dhaka, asserting: 'It is not ethical that, in 
some clinics of this area, the doctors and trainees of the Government Medical College see patients in between office hours',

One common strategy to overcome doctor shortages during daytime hours was the use of on-call doctors. These doctors typically practice in public sector facilities in close proximity to a private sector clinic that relies on their services and will respond to calls when needed. As one clinic owner (and provider) from Sylhet explained: 'Within a few minutes we come to see the patients. Within five to ten minutes the specialist also comes here. If we are informed we come here from anywhere. Or another specialist comes to manage everything'.

This strategy was popular among private clinic owners given its perceived cost savings over standard practices of recruiting and paying the salaries of three doctors to cover a 24-hour service or having to hire specialist doctors full time when their services are not always needed. It was also noted that the strategy was not without hazards. Given that many private clinics rely on commonly performed surgeries (C-sections and appendectomies) to ensure financial sustainability, in the context of life-threatening complications, reliance on on-call doctors who are located off-site may substantially heighten risk to patients.

Recruiting and retaining qualified nurses was also identified as a major challenge by many private sector respondents. As a result, nursing care was often provided by unqualified or untrained persons such as cleaners and ayas who are meant to provide non-technical caregiving support to patients and their families. According to private sector respondents, the reliance on unqualified personnel was due to the dearth of qualified nurses on the market and the high salaries they command. As one clinic owner from Dhaka despaired:

It is impossible for me to keep 6 nurses (on staff); it is not possible for any clinic to give 60000 taka (USD 711.57) for their salary (10000 taka each, USD 118.59). Maybe it is possible for (large private hospitals like) Apollo and Square, but not for me. (Instead) we must hire secondary school certificate girls and train them on the job...

In circumstances when a private sector clinic is unable to handle a complicated case due to lack of capacity, referral to public hospitals and medical colleges was justified. Respondents noted this practice was especially common among patients requiring specialty care or those with emergent, deteriorating or potentially fatal health conditions. Several providers further noted the reputational risk in being held responsible for a patient's death and hence the reliance on referral as a strategy to avoid potential fatalities that might damage their professional reputation or that of the facility in which they work. Referral shifted responsibility for potential accusations of malpractice to the receiving facility, which most commonly was the city's public hospital. As one private sector owner/provider from Khulna admitted, 'We don't take the risk of keeping critical patients. They are referred to the
Government Hospital, where there are ICU facilities, or to larger private hospitals based on their (financial) ability'.

This widespread practice highlights broader limitations in critical care capacity within urban areas that need to be addressed. ${ }^{28}{ }^{29}$ It can also exacerbate the vulnerability of the urban poor, as the referral destination is often determined based on ability to pay. Even if a private hospital is closest, many providers stated that they were more apt to refer poorer patients to public facilities or medical colleges, while better-off patients were sent to closer private facilities. Patient's desires, frequently motivated by perceived quality of services, was a further factor influencing referral patterns. More virtuous behaviour was also reported. Some providers stated that their referral decisions were based on the quality of care provided at the receiving facility, and several claimed that instead of taking commissions from referral facilities, they asked that patient discounts be provided instead.

Interestingly, almost all of the respondents in the study acknowledged that the absence of a formalised referral system contributed to poor health outcomes and described the challenge of patients arriving too late for effective treatment. Suggestions were also made that a formal referral system be implemented to assist primary providers in ensuring patients be directed to appropriate levels of care.

\section{DISCUSSION}

This exploratory study is among the first in Bangladesh to query the underlying motivations and strategies of the urban private for-profit sector and their implications for healthcare quality and accessibility. However, certain limitations must be acknowledged. The most challenging of these was the reluctance of some private sector respondents to divulge details about the strategies they employ to grow their business. In other instances, respondents may have constructed their replies in a socially desirable manner to mitigate judgement about the business strategies they employ, especially if unethical or informal practises were revealed. Although efforts were made to maximise trust by approaching respondents through personal contacts and social networks, richer and more trustworthy data might have been produced had a lengthier period of rapport building been possible. While a risk of selection bias was inherent in our approach, this was justified given our concern that private sector respondents would not divulge their business strategies to strangers. Selection bias may also have occurred in exit interviews as clients may have been more inclined to give a positive evaluation of the quality of care received at the time of discharge.

An interesting first insight emerging from analysis was the complexity of motivations prompting involvement in the private for-profit sector. IDIs with owners, managers and providers challenged widespread perceptions that financial interests are the singular driving force for engagement in private healthcare business. Rather, public 
service, social status and family obligation figured prominently alongside profit and financial security as factors motivating participation. Leveraging these non-financial motivations might serve to further encourage social responsibility in the sector or potential participation in health financing schemes that aim to increase affordable healthcare access to the urban poor. That being said, private sector engagement is ultimately contingent on success in turning a profit. ${ }^{30}$ In other words, the scope for market expansion must be sufficient to support lower per patient revenues typically associated with fee for service and prepayment schemes. ${ }^{31-33}$

In this paper, the analysis of strategies was usefully structured around the BPM. ${ }^{24}{ }^{25}$ As the model specifies, in order to deliver a profitable value proposition, private healthcare actors must employ strategies that enable a best fit between the products or services offered, the market environment and their organisation's capabilities. In this discussion, we consider each of these components to gain insight about the complex ecosystem in which the urban private sector is located and the manner in which business interests are pursued within a competitive healthcare market. Of particular interest is how the strategies employed are conducive to achieving greater efficiency, equity or scale and their implications for Bangladesh's broader policy goals in support of UHC.

As regards products and services, there was a near-consensus viewpoint among study respondents about the necessity of providing patient-friendly services and making patients 'happy'. These objectives appear to be backed up by a range of 'patient-loyalty' strategies that included 'good' provider behaviour, the discretionary use of discounts on consultation fees, drugs and procedures and offering extended service hours convenient to the working population. Interestingly, however, exit interviews with patients revealed that consultation time was only slightly longer than public sector facilities and did not appear to be an important factor influencing patient perceptions of quality. ${ }^{34}$ These results correspond with global evidence that patients are willing to pay for private healthcare if they perceive providers are respectful and responsive to their needs ${ }^{35} 36$ and that good provider-patient relations increase the likelihood of sustained treatment seeking, ${ }^{37} 38$ and attracting new clientele. Indeed, adapting service pricing and delivery modalities to the needs and preferences of healthcare customers is emblematic of private sector practice globally. ${ }^{39}$ This behaviour can be explained by the aspiration to gain and sustain market share through customer loyalty when other private sector actors or NGOs may be offering lower cost services.

To ensure their position in a crowded healthcare market, many private sector respondents indicated their reliance on brokers and agents. Referral fees paid to these middlemen ensured a fresh client flow and competitive advantage. Among those 'captured' were patients diverted from government facilities where services are free. Very similar was the widespread practice of offering referral fees to doctors who direct their patients to preferred private sector clinics. ${ }^{30}$ In both cases, these practices are only effective market strategies if the costs incurred amplify profit. It was unclear, however, whether such calculations were made, and the extent to which they end up being subsidised by patient out-of-pocket expenditures. $^{7}$

The role of pharmaceutical agents in shaping the market environment and the prescription practices of private sector doctors was also widely acknowledged. Many private sector respondents valued the incentives that they receive from pharmaceutical companies, some of which are passed onto patients, such as discounted prices on 'free' medicine samples. A few others expressed concerns that the medicines pushed by pharmaceutical representatives were expensive or unknown in terms of efficacy. The adverse influence of pharmaceutical incentives on private sector business has been emphasised by others, ${ }^{40-42}$ and it is well recognised that aggressive marketing strategies undermine patient safety and ethical medical conduct and need firm regulation. ${ }^{40-44}$

Capacity constraints related to human resources, especially of nursing staff and specialist doctors, were almost universally identified by private sector respondents. At the same time, concerns related to the costs of keeping full-time staff were also acknowledged. A variety of workaround strategies were reported to overcome gaps and minimise costs such as the use of on-call specialists from the public sector, reliance on doctors in training or recent medical graduates, as well as filling sector-wide short-falls in the number of nurses through onsite and unregulated nurse training to unqualified personnel. The implications of these adaptive mechanisms in terms of quality and costs to the public health system and patient safety warrant assessment. Moreover, these strategies provide insights into some of drivers of the widespread phenomena of 'dual practice' that many health systems struggle to manage from cost, quality and accountability perspectives. ${ }^{13} 304546$

Other strategies employed to overcome capacity limitations included the referral of complicated cases to public sector tertiary facilities. Several respondents noted that the absence of a formal urban referral system inclusive of both private and public sectors heightens patient risk as inappropriate or late referrals may result. The development of a system that identifies the fastest and safest route to appropriate critical care services, whatever the location, represents a critical area for policy attention. This includes the transfer of patient information so that expensive diagnostic tests are not needlessly repeated, ${ }^{47}$ enhanced capacity for first aid services to ensure that patients are stabilised during transport and the availability of proximate and effective ambulatory services.

Although our study was focused on so-called 'formal' small to medium-sized private health facilities in urban Bangladesh, striking was the degree to which 'informality' characterised most aspects of their business model ranging from the way prices were levied, health workers deployed 
and business hours set. Not a single respondent indicated the existence of a written business plan or even standard operating procedures to guide the day-to-day or longer term development of services. Similarly lacking was a formal or standardised system of reporting on the volume, quality or costs of services provided. Paradoxically, 'professional reputation'-subjectively or tacitly determined by the 'public'was noted as critical to sustaining a successful healthcare business. In short, we observed a variety of stopgap strategies that align services, market environment and are illustrative of a sector that is getting by but with little apparent emphasis on achieving greater efficiency or scale.

This qualitative exploration of the motivations and business strategies of those involved in small and medium-sized urban private for-profit healthcare delivery offers insight on how this sector might be harnessed more effectively towards the broader national policy aims of UHC. Features of the sector that represent strengths or positive assets include professed motivations of service, patient-centredness and responsiveness, innovative approaches to pricing, sensitivity to differential ability to pay and the desire to maintain a professional reputation within the healthcare market. These features have established the sector's legitimacy and dominance in the urban context and account for its popularity as a source of care. ${ }^{303945}$ They also align to some degree with UHC goals of affordable access to quality healthcare without risk of financial harm.

At the same time, certain adverse practices exist that are contrary to Bangladesh's aspirations for UHC. ${ }^{48}$ Patient brokers and pharmaceutical incentives are likely to lead to oversupply of services and overprescription that do not match with needs and may promote public health threats such as antimicrobial resistance ${ }^{49}$ Furthermore, staffing models that rely on part-time, junior and unregulated training pose serious problems with quality and safety of services. From an affordability perspective, accessibility among the poorest segments of the population is unclear, and the pay-per-service model of provision is likely to strain household budgets and push significant numbers into poverty. ${ }^{5051}$

Given the size and centrality of this sector to urban health in Bangladesh., ${ }^{2023}$ the policy conundrum becomes how best to amplify strengths and shore-up shortfalls of this important segment of the urban health system. Perhaps the most challenging attribute of the sector is its inherent informality in a broader health systems context that is also characterised by weak governance, particularly in urban areas. This context argues against sweeping de jure regulatory reforms on multiple fronts as they are highly unlikely to be implemented in any meaningful way. Rather, more discrete, focused efforts on specific parts of the sector, that is, pharmaceutical prescription practices that engage the principal actors in changing behaviours, may be more effective in nudging the private sector more towards the goals of UHC.

While our findings are appropriately contextualised for urban Bangladesh, they also resonate with other low-income and middle-income countries characterised by increasing urban healthcare demand, a growing private sector and a weak regulatory environment. ${ }^{52}$ However, the need remains for nuanced ethnographic work that examines the particularities of a highly diverse sector and the unique manner in which products, markets and capacity are aligned to sustain successful business. ${ }^{53}$ Understanding these complexities and the larger ecosystem in which the private sector operates will lend itself to policies that are fit for purpose and effective in harnessing supply and ensuing quality and affordable access to the urban poor.

\section{CONCLUSION}

In urban Bangladesh, the private for-profit sector plays a crucial role in meeting a growing demand for healthcare in a context of limited public provision. Within this massive, heterogeneous yet predominantly informal range of providers, small and medium-sized private clinics and hospitals are important purveyors of so-called 'formal' healthcare services. Focusing on the motivations and strategies undergirding urban private healthcare business, our findings confirm prevailing assumptions about the sector's profit orientation, informality and sometimes deleterious practices. At the same time, certain strategies yield benefits to healthcare consumers like the sector's emphasis on responsive, patient-friendly services. Given the weak regulatory capacity of national and local authorities and professional associations alike, the provision of incentives that promote greater accountability within the private for-profit sector and reward efforts to increase the affordability and quality of services may be a more realistic strategy towards UHC. Support in extending the private sector's largely curative focus to include preventive and promotive services is also critical given the lacunae of primary care services in urban areas. Most importantly, policies that support UHC within the realities of a highly pluralistic health market must accommodate the financial interests of this massive, diverse and growing private sector. Policies and programmes that encourage private sector quality and effectiveness, and enable even greater market share, may function to drive out the subset of private sector players whose business model relies on overcharging, oversupplying or providing substandard care.

Acknowledgements This research study was funded by Department for International Development (DFID). icddr,b acknowledges with gratitude the commitment of DFID to its research efforts. icddr,b is also grateful to the Governments of Bangladesh, Canada, Sweden and the UK for providing core/ unrestricted support. Authors also recognise the contribution of all respondents for their participation in the study and the valued engagement of Zubair Akhtar, Gladys Leterme and Razib Mamun in initial data collection and analysis.

Contributors Criteria \#1: substantial contributions to the conception or design of the work, or the acquisition, analysis or interpretation of data: all authors. Criteria \#2: drafting the work or revising it critically for important intellectual content: AMA and RA drafted the manuscript. TAS, SSY, SA and IA reviewed critically for important intellectual content. AMA and RA finally revised the version submitted with inputs from all other coauthors. Criteria \#3: final approval of the version published: all authors. Criteria \#4: agreement to be accountable for all aspects of the work in 
ensuring that questions related to the accuracy or integrity of any part of the work are appropriately investigated and resolved: all authors.

Funding Funding was received by AMA from DFID for this work; grant number: 01020.

Competing interests None declared.

Patient consent for publication Not required.

Ethics approval The project was approved by the Research Review Committee and Ethical Review Committee of icddr,b.

Provenance and peer review Not commissioned; externally peer reviewed.

Data sharing statement The data sets analysed in the current study are not publicly available but are available from the Ethical Review Committee of icddr,b on reasonable request after meeting the necessary criteria for access to confidential data.

Open access This is an open access article distributed in accordance with the Creative Commons Attribution Non Commercial (CC BY-NC 4.0) license, which permits others to distribute, remix, adapt, build upon this work non-commercially, and license their derivative works on different terms, provided the original work is properly cited, appropriate credit is given, any changes made indicated, and the use is non-commercial. See: http://creativecommons.org/licenses/by-nc/4.0/.

\section{REFERENCES}

1. Adams AM, Ahmed SM, Evans TG. Universal health care in Bangladesh-promises and perils. Lancet Glob Health 2018;6:e10-11.

2. Ahmed I. Building Resilience of Urban Slums in Dhaka, Bangladesh. Procedia - Soc Behav Sci 2016;218:202-13.

3. United Nations. World Urbanization Prospects: The 2014 Revision, Highlights. Department of Economic and Social Affairs, Population Division. 2014.

4. United Nations. World Urbanization Prospects: the 2018 revision, key facts: Department of Economic and Social Affairs, Population Division, 2018.

5. World Health Organization. Strengthening the capacity of governments to constructively engage the private sector in providing essential health-care services. 27: World Health Assembly Resolution WHA63, 2010.

6. Sengupta U, Doll CNH, Gasparatos A, et al. Sustainable smart cities: applying complexity science to achieve Urban Sustainability [Policy Brief No. 12]. Tokyo, Japan: Institute for the Advanced Study of Sustainability. United Nations University 2017.

7. Health Economics Unit, Ministry of Health and Family Welfare. Bangladesh National Health Accounts 1997-2015: preliminary results. Dhaka: Health Economics Unit, Ministry of Health and Family Welfare, 2015.

8. Chowdhury AM, Bhuiya A, Chowdhury ME, et al. The Bangladesh paradox: exceptional health achievement despite economic poverty. Lancet 2013;382:1734-45.

9. El Arifeen S, Christou A, Reichenbach L, et al. Community-based approaches and partnerships: innovations in health-service delivery in Bangladesh. Lancet 2013;382:2012-26.

10. Houweling TA, Ronsmans C, Campbell OM, et al. Huge poor-rich inequalities in maternity care: an international comparative study of maternity and child care in developing countries. Bull World Health Organ 2007;85:745-54.

11. Bennett S, McPake B. Private health providers in developing countries: serving the public interest. Zed books: The University of Chicago Press, 1997.

12. McPake B, Hanson K. Managing the public-private mix to achieve universal health coverage. Lancet 2016;388:622-30.

13. Ahmed SM, Evans TG, Standing $\mathrm{H}$, et al. Harnessing pluralism for better health in Bangladesh. Lancet 2013;382:1746-55.

14. National Institute of Population Research and Training (NIPORT); International Centre for Diarrhoeal Disease Research, Bangladesh (icddr,b); and MEASURE Evaluation. Bangladesh Maternal Mortality and Health Care Survey 2016: Preliminary Report. Dhaka, Bangladesh, and Chapel Hill, NC, USA: NIPORT, icddr,b, and MEASURE Evaluation, 2017.

15. National Institute of Population Research and Training (NIPORT), Mitra and Associates, and ICF International. Bangladesh Demographic and Health Survey 2011. Dhaka, Bangladesh and Calverton, Maryland, USA: NIPORT, Mitra and Associates, and ICF International, 2013.

16. National Institute of Population Research and Training (NIPORT), Mitra and Associates, and ICF International. Bangladesh
Demographic and Health Survey 2014: Key Indicators. Dhaka, Bangladesh, and Rockville, Maryland, USA, 2015.

17. Ahmed SM, Hossain MA, Rajachowdhury AM, et al. The health workforce crisis in Bangladesh: shortage, inappropriate skill-mix and inequitable distribution. Hum Resour Health 2011;9:3.

18. Islam R, Hossain S, Bashar F, et al. Contracting-out urban primary health care in Bangladesh: a qualitative exploration of implementation processes and experience. Int $J$ Equity Health 2018:17:93.

19. Govindaraj R, Raju D, Secci F, et al. Health and Nutrition in Urban Bangladesh: Social determinants and health sector governance. Directions in Development-Human Development. 131. Washington, DC: World Bank, 2018.

20. Adams AM, Ahmed S, Hasan SM, et al. Mapping the Urban Healthcare Landscape in 5 City Corporations. Bangladesh, Dhaka: icddr,b, 2015.

21. Management Information System. Directorate General of Health Services. Bangladesh Health Bulletin 2007. Ministry of Health and Family Welfare. Government of the People's Republic of Bangladesh: Dhaka, Bangladesh, 2007.

22. Management Information System. Directorate General of Health Services. Bangladesh Health Bulletin 2017. Ministry of Health and Family Welfare. Government of the People's Republic of Bangladesh: Dhaka, Bangladesh, 2017.

23. Adams AM, Ahmed T, Islam R, et al. Mapping health facilities in Sylhet City Corporation, Bangladesh. Dhaka, Bangladesh: Deutsche Gesellschaft fürlnternationale Zusammenarbeit (GIZ) GmbH, 2014.

24. Christensen CR, Kenneth RA, Joseph LB, et al. Business Policy: Text and Cases. 5th edn. Homewood, IL: Richard D. Irwin, 1982.

25. Alford J, Greve C. Strategy in the public and private sectors: similarities, differences and changes. Adm Sci 2017;7:35.

26. Ritchie J, Lewis J. Qualitative research practice: a guide for social science students and researchers. London: Sage, 2003.

27. Code of Pharmaceutical Marketing Practices (CPCM). Directorate General of Drug Administration. Ministry of Health and Family Welfare. Government of the People's Republic of Bangladesh. 1992 https://www.dgda.gov.bd/index.php/2013-03-31-05-16-29/forms/77code-of-pharamaceutical-marketion-practices/file.

28. James P Grant School of Public Health. Bangladesh Health Watch Report 2014. Urban Health Scenario: looking beyond 2015. Dhaka, Bangladesh: James P Grant School of Public Health, BRAC University, 2015.

29. Adams AM, Islam R, Ahmed T. Who serves the urban poor? A geospatial and descriptive analysis of health services in slum settlements in Dhaka, Bangladesh. Health Policy Plan 2015;30(suppl 1):i32-45.

30. Wadge $\mathrm{H}$, Roy $\mathrm{R}$, Sripathy $\mathrm{A}$, et al. Evaluating the impact of private providers on health and health systems. London, UK: Imperial College London, 2017.

31. Sood N, Wagner Z. Social health insurance for the poor: lessons from a health insurance programme in Karnataka, India. BMJ Glob Health 2018;3:e000582.

32. Bhattacharyya O, Khor S, McGahan A, et al. Innovative health service delivery models in low and middle income countries - what can we learn from the private sector? Health Res Policy Syst 2010;8:24.

33. Escobar ML, Griffin CC, Shaw RP. The impact of health insurance in low-and middle-income countries. Washington, DC: Brookings Institution Press, 2010.

34. Bank W. Comparative advantages of public and private health care providers inBangladesh. Dhaka, Bangladesh: World Bank, 2005.

35. Birungi $\mathrm{H}$. Injections and self-help: risk and trust in Ugandan health care. Soc Sci Med 1998;47:1455-62.

36. Russell S. Treatment-seeking behaviour in urban Sri Lanka: trusting the state, trusting private providers. Soc Sci Med 2005;61:1396-407.

37. Mechanic D, Meyer S. Concepts of trust among patients with serious illness. Soc Sci Med 2000;51:657-68.

38. Straten GF, Friele RD, Groenewegen PP. Public trust in Dutch health care. Soc Sci Med 2002;55:227-34.

39. Tung E, Bennett S. Private sector, for-profit health providers in low and middle income countries: can they reach the poor at scale? Global Health 2014;10:52.

40. Mohiuddin M, Rashid SF, Shuvro Ml, et al. Qualitative insights into promotion of pharmaceutical products in Bangladesh: how ethical are the practices? BMC Med Ethics 2015;16:80.

41. Shah SA, Khawaja HA. Unethical marketing practices of pharmaceutical companies in Pakistan: a case study of Sukkur division. Handbook on the Economic, Finance and Management Outlooks, 2013.

42. Roy N, Madhiwalla N, Pai SA. Drug promotional practices in Mumbai: a qualitative study. Indian J Med Ethics 2007;4:57-61. 
43. Ahmed RR, Saeed A. Pharmaceutical drug promotion practices in Pakistan: Issues in ethical and non-ethical pharmaceutical practices. Middle-East J Scientific Res 2014;20:1630-40.

44. Mandal BK, Yadav SK, Abhishek K, et al. Relationship between doctors and pharmaceutical industry: An ethical perspective. Int $J$ Pharmaceut Biological Arch 2012;3:415-7.

45. Morgan R, Ensor T, Waters H. Performance of private sector health care: implications for universal health coverage. Lancet 2016;388:606-12.

46. Hipgrave DB, Hort K. Dual practice by doctors working in South and East Asia: a review of its origins, scope and impact, and the options for regulation. Health Policy Plan 2014;29:703-16.

47. Basu S, Andrews J, Kishore S, et al. Comparative performance of private and public healthcare systems in low- and middle-income countries: a systematic review. PLoS Med 2012;9:e1001244.

48. Joarder T, Chaudhury TZ, Mannan I. Universal Health Coverage in Bangladesh: activities, challenges, and suggestions. Adv Public Health 2019; Vol 2019:1-12
49. Omulo S, Thumbi SM, Lockwood S, et al. Evidence of superficial knowledge regarding antibiotics and their use: Results of two crosssectional surveys in an urban informal settlement in Kenya. PLOS One 2017;12:e0185827.

50. Islam MR, Rahman MS, Islam Z, et al. Inequalities in financial risk protection in Bangladesh: an assessment of universal health coverage. Int J Equity Health 2017;16:59.

51. Rahman MM, Gilmour S, Saito E, et al. Health-related financial catastrophe, inequality and chronic illness in Bangladesh. PLoS One 2013;8:e56873.

52. Pettigrew LM, Mathauer I. Voluntary health insurance expenditure in low-and middle-income countries: Exploring trends during 19952012 and policy implications for progress towards universal health coverage. Int J Equity Health 2016;15:67.

53. Ahlin T, Nichter M, Pillai G. Health insurance in India: what do we know and why is ethnographic research needed. Anthropol Med 2016;23:102-24. 